\title{
Optical identifications of High Frequency Peakers ${ }^{\star}$
}

\author{
D. Dallacasa ${ }^{1,2}$, R. Falomo ${ }^{3}$, and C. Stanghellini ${ }^{4}$ \\ 1 Dipartimento di Astronomia, Università degli Studi, via Ranzani 1, 40127 Bologna, Italy \\ 2 Istituto di Radioastronomia - CNR, via Gobetti 101, 40129 Bologna, Italy \\ 3 Osservatorio Astronomico, Vicolo dell'Osservatorio 5, 35122 Padova, Italy \\ ${ }^{4}$ Istituto di Radioastronomia - CNR, C.P. 141, 96017 Noto (SR), Italy
}

Received 21 September 2001 / Accepted 8 November 2001

\begin{abstract}
We present CCD observations of 13 objects from a complete sample of 55 bright High Frequency Peaker (HFP) radio sources, and provide optical identification for 12 of them. Images in $R$ and $V$ filters have been used to derive some additional information concerning the host of the radio source. Three hosts are likely to be galaxies, one resulted slightly extended, while the remaining 8 are likely distant quasars. Based on these identifications and those available in the literature, we find that the fraction of quasars in our HFP sample is significantly higher than in samples of Compact Steep-Spectrum and GHz-Peaked Spectrum radio sources.
\end{abstract}

Key words. galaxies: active - radio continuum: galaxies - quasars: general

\section{Introduction}

The population of intrinsically small and bright radio sources has been investigated in the past by studying samples of Compact Steep Spectrum (CSS) and GHz-Peaked Spectrum (GPS) radio sources (see Spencer et al. 1989; Fanti et al. 1990, 1995; Stanghellini et al. 1998; O'Dea 1998). These sources are characterized by optically thin steep radio emission with a turnover occurring at frequencies ranging from about $100 \mathrm{MHz}(\mathrm{CSSs})$ to about $1 \mathrm{GHz}$ (GPSs). When the spectral peak is found at frequencies higher than a few $\mathrm{GHz}$, the source is classified High Frequency Peaker (HFP, Dallacasa et al. 2000).

There is a substantial absorption of the low frequency radiation, but it is unclear whether it is due either to synchrotron self-absorption (SSA) favoured by Fanti et al. (1995) and Snellen et al. (2000) or to free-free absorption (FFA) as proposed by Bicknell et al. (1997). It is also possible that both mechanisms play a role.

The optical identifications in all the forementioned samples are about evenly divided into galaxies, typically in the redshift range between 0.1 and 1 , and quasars, generally at higher redshift, between about 0.8 and 3.5. This

Send offprint requests to: D. Dallacasa,

e-mail: ddallaca@ira.bo.cnr.it

* Based on observations made with the Italian Telescopio Nazionale Galileo (TNG) operated on the island of La Palma by the Centro Galileo Galilei of the CNAA (Consorzio Nazionale per l'Astronomia e l'Astrofisica) at the Spanish Observatorio del Roque de los Muchachos of the Instituto de Astrofisica de Canarias. implies that quasars have on average a higher intrinsic turnover frequency.

It has been statistically found that a fraction of CSS quasars has apparent sub-galactic size due to projection effects (Fanti et al. 1990), and further, the pc-scale radio morphology seems to distinguish between galaxies (compact doubles or Compact Symmetric Objects, CSOs) dominated by mini-lobe emission, and quasars, where most of the radio emission comes from unresolved cores and pc-scale jets (e.g. Stanghellini et al. 1997). There are a few exceptions to this picture, and those particularly interesting are the lobe dominated quasars. In general the radio cores of the GPS quasars are less prominent than in flat spectrum quasars (Stanghellini et al. 2001). All this is consistent with Unified Scheme predictions.

The currently most accepted model (known as the "youth model") to interpret these intrinsically small radio sources postulates that they are indeed young objects still developing/deploying their radio emission within the host galaxy, and their fate is to grow up to become extended sources (hundreds of kpc in size), stage where they spend most of their life (Fanti et al. 1995; Readhead et al. 1996), progressively decreasing their radio power by about one order of magnitude. A self-similar evolutionary model for CSS/GPS radio galaxies has been proposed by Snellen et al. (2000) using a slightly different parametrization.

The alternative model (the "frustration model") suggests that the radio emitting regions would never expand due to a particularly dense medium impeding the expansion of the radio lobes (e.g. van Breugel 1984; Baum et al. 1990). 
Table 1. HFP sources observed at the TNG. Columns list the name (1), the radio position (2, 3 Right Ascension and Declinations respectively, J2000.0), the total exposure time in seconds in the $B(4), V(5), R(6)$ and $I$ (7) filters respectively.

\begin{tabular}{clllrrc}
\hline \hline $\begin{array}{c}\text { Source name } \\
(1)\end{array}$ & $\begin{array}{c}\text { RA } \\
(2)\end{array}$ & \multicolumn{1}{c}{$\begin{array}{c}\text { DEC } \\
(3)\end{array}$} & $\begin{array}{c}B \\
(4)\end{array}$ & $\begin{array}{r}V \\
(5)\end{array}$ & $\begin{array}{c}R \\
(6)\end{array}$ & $\begin{array}{c}I \\
(7)\end{array}$ \\
& $\mathrm{h} \mathrm{m} \mathrm{s}$ & \multicolumn{1}{c}{${ }^{\prime \prime}$} & $\mathrm{s}$ & $\mathrm{s}$ & \multicolumn{1}{c}{$\mathrm{s}$} & \\
\hline \hline $\mathrm{J} 0003+2129$ & 000319.3497 & 212944.436 & - & 1800 & 1890 & - \\
$\mathrm{J} 0037+0808$ & 003732.15 & 080812.6 & - & 1200 & 1200 & - \\
$\mathrm{J} 0329+3510$ & 032915.3553 & 351005.940 & 180 & 180 & 180 & 180 \\
$\mathrm{~J} 0357+2319$ & 035721.6088 & 231953.859 & 300 & 300 & 900 & - \\
$\mathrm{J} 0428+3259$ & 042805.8126 & 325951.975 & - & 600 & 900 & - \\
$\mathrm{J} 1811+1704$ & 181143.1843 & 170457.279 & - & 1200 & 1200 & - \\
$\mathrm{J} 1855+3742$ & 185527.7057 & 374256.986 & - & 1200 & 1200 & - \\
$\mathrm{J} 2021+0515$ & 202135.2802 & 051504.770 & - & 600 & 900 & - \\
$\mathrm{J} 2024+1718$ & 202456.5638 & 171813.208 & 60 & 30 & 60 & - \\
$\mathrm{J} 2114+2832$ & 211458.3340 & 283257.206 & 600 & 600 & 600 & 600 \\
$\mathrm{~J} 2203+1007$ & 220330.9534 & 100742.584 & - & 1800 & 1950 & - \\
$\mathrm{J} 2207+1652$ & 220752.8674 & 165217.837 & 180 & 180 & 180 & - \\
$\mathrm{J} 2212+2355$ & 221205.9680 & 235540.591 & 180 & 180 & 180 & - \\
\hline \hline
\end{tabular}

So far, various observations across the whole electromagnetic spectrum failed to reveal the presence of such particularly dense medium, suggesting, otherwise, that the host galaxies of these compact sources share the same properties of the hosts of extended radio sources (e.g. Fanti et al. 2000).

Further, the observation of proper motions of the outer edges (hot-spots) in Compact Symmetric Objects (CSOs), a subclass of CSS/GPS radio sources, discovered by Owsianik et al. (1998) definitely support the youth model. Nowadays, proper motions with apparent velocities of $0.2-0.4 c$ have been discovered in about ten sources (Fanti 2000).

The radiative ages estimated for these small sources are consistent with the hypothesis that they are young (Murgia et al. 1999).

There is a correlation between the turnover frequency and the projected linear size (i.e. age), and the youngest (i.e. the smallest) objects have the highest turnover frequencies (e.g. O'Dea 1998); the peak in the radio spectrum moves at lower frequencies as the source expands and the energy density decreases. Searching for newly born radio sources means to look for radio spectra peaking at a few $\mathrm{GHz}$ or higher frequencies. In principle, the higher the turnover frequency the younger the object.

\section{The bright HFP sample}

To search for very young radio sources we are investigating a sample of 55 bright High Frequency Peakers (HFPs) selected by comparing the NRAO VLA Sky Survey, NVSS, (Condon et al. 1998) and the Green Bank, 87GB, (Gregory et al. 1996) radio catalogues at 1.4 and $4.9 \mathrm{GHz}$, respectively. Details on the selection procedure and on the final HFP source list can be found in Dallacasa et al. (2000).

We used the NASA/IPAC Extragalactic Database (NED) to investigate the current identification and redshift status of the 55 bright HFP sources. This search provided the following information: 29 already have both optical identification and redshift. Of these 24 are quasars, 1 is classified as "stellar" with an uncertain redshift, and therefore likely to be also a quasar, 3 are galaxies (one of them is a broad line galaxy) and the remaining object is a BL Lac. Further, either in the literature or on the red digitized Palomar Observatory Sky Survey POSS-I images 5 have "stellar" appearance, 1 is a BL Lac without redshift and 4 more are likely to be galaxies. Finally 16 are Empty Fields (EF); the summary of the previous status of the optical identification for the whole sample is given in Dallacasa et al. (2000). We note that a few sources appearing as empty fields in the POSS-I plates have a weak counterpart in the POSS-II.

\section{The optical data}

On September 1st and 2nd 2000, we observed 13 HFP sources from the bright sample with the Device Optimized for the LOw RESolution (DOLORES) on the $3.6 \mathrm{~m}$ Telescopio Nazionale Galileo (TNG) located in La Palma (Canary Islands, Spain). The instrument was used in imaging mode, operating a Loral thinned and back-illuminated $2048 \times 2048$ CCD, with a scale of $0.275 \mathrm{arcsec} / \mathrm{px}$, yielding a field of view of about $9.4 \times$ 9.4 arcmin.

All the sources listed in Table 1 were observed in $R$ and $V$ filters. The exposure times were initially estimated on the basis of the appearance of the field in the digitized POSS-I images. The sources with a clear counterpart in such fields were also observed with the $B$ filter, while for two of them also the $I$ frame was acquired. Table 1 reports the total exposure time per filter. $R$ and $V$ filters are in the Cousins system.

Five out of 13 sources (J0329+2129, J2024+1718, $\mathrm{J} 2114+2832$, J2207+1652 and J2212+2355) have a counterpart in the digitized POSS-I and/or POSS-II images, 
Table 2. Optical positions and magnitudes from TNG observations: the various columns report the name (1), Right Ascension (2) and Declination (3) in J2000.0, differences between the optical and radio positions in arcsecond, $B$ (6), $V(7)$, $R(8)$ and $I(9)$ magnitudes, and $V-R(10)$, optical morphology (11), for resolved (R) or unresolved (U) objects; when a "?" is present, the classification is uncertain.

\begin{tabular}{|c|c|c|c|c|c|c|c|c|c|c|}
\hline \multirow[t]{2}{*}{$\begin{array}{c}\text { Source Name } \\
\text { (1) }\end{array}$} & $\begin{array}{l}\text { RA } \\
(2)\end{array}$ & \multirow{2}{*}{$\begin{array}{c}\text { DEC } \\
(3) \\
\circ \quad, \quad \prime \prime\end{array}$} & \multirow{2}{*}{$\begin{array}{c}\Delta_{\mathrm{RA}} \\
(4) \\
\prime \prime\end{array}$} & \multirow{2}{*}{$\begin{array}{c}\Delta_{\mathrm{DEC}} \\
(5) \\
\prime \prime\end{array}$} & \multirow[t]{2}{*}{$\begin{array}{c}B \\
(6)\end{array}$} & \multirow[t]{2}{*}{$\begin{array}{l}V \\
(7)\end{array}$} & \multirow[t]{2}{*}{$\begin{array}{c}R \\
(8)\end{array}$} & \multirow[t]{2}{*}{$\begin{array}{c}I \\
(9)\end{array}$} & \multirow[t]{2}{*}{$\begin{array}{c}-R \\
(10)\end{array}$} & \multirow[t]{2}{*}{$\begin{array}{c}\text { Opt. Morph. } \\
(11)\end{array}$} \\
\hline & $\mathrm{h} \mathrm{m} \mathrm{s}$ & & & & & & & & & \\
\hline $\mathrm{J} 0003+2129$ & 000319.360 & 212943.83 & 0.15 & -0.61 & - & 20.58 & 19.65 & - & 0.93 & $\mathrm{R}$ \\
\hline $\mathrm{J} 0037+0808$ & unidentified & unidentified & - & - & - & $>24.5$ & $>24.5$ & - & $\ldots$ & $\ldots$ \\
\hline $\mathrm{J} 0329+3510$ & 032915.313 & 351005.91 & 0.52 & -0.03 & 16.26 & 16.06 & 16.06 & 15.74 & 0.00 & $\mathrm{U}$ \\
\hline $\mathrm{J} 0357+2319$ & 035721.635 & 231954.28 & 0.36 & 0.42 & 18.82 & 18.23 & 17.76 & - & 0.47 & $\mathrm{U}$ \\
\hline J0428+3259 & 042805.867 & 325952.35 & 0.68 & 0.38 & - & 20.23 & 19.25 & - & 0.98 & $\mathrm{R}$ \\
\hline $\mathrm{J} 1811+1704$ & 181143.176 & 170457.31 & -0.12 & 0.03 & - & 20.52 & 20.03 & - & 0.49 & $\mathrm{U}$ \\
\hline $\mathrm{J} 1855+3742$ & 185527.638 & 374256.64 & -0.80 & -0.35 & - & 21.11 & 20.70 & - & 0.41 & $\mathrm{R}$ ? \\
\hline $\mathrm{J} 2021+0515$ & 202135.297 & 051504.85 & 0.25 & 0.08 & - & 20.70 & 21.08 & - & -0.38 & $\mathrm{U}$ \\
\hline $\mathrm{J} 2024+1718$ & 202456.571 & 171813.52 & 0.10 & 0.32 & 18.06 & 17.64 & 17.55 & - & 0.09 & $\mathrm{U}$ \\
\hline $\mathrm{J} 2114+2832$ & 211458.348 & 283257.26 & 0.18 & 0.05 & 18.70 & 18.38 & 18.35 & 17.93 & 0.03 & $\mathrm{U}$ \\
\hline $\mathrm{J} 2203+1007$ & 220330.932 & 100742.81 & 0.04 & -0.16 & - & 23.53 & 22.28 & - & 1.25 & $\mathrm{R}$ \\
\hline $\mathrm{J} 2207+1652$ & 220752.843 & 165217.75 & -0.35 & -0.09 & 20.25 & 20.27 & 19.91 & - & 0.36 & $\mathrm{U}$ \\
\hline $\mathrm{J} 2212+2355$ & 221205.978 & 235540.79 & 0.14 & 0.20 & 18.21 & 19.17 & 18.70 & - & 0.47 & $\mathrm{U}$ \\
\hline
\end{tabular}

generally of stellar appearance, although a proper classification is not possible, given that they are rather weak.

The radio positions in Table 1 are taken from the Jodrell Bank - VLA Astrometric Survey (JVAS) (Patnaik et al. 1992; Browne et al. 1998; Wilkinson et al. 1998) and are very accurate. J0037+0808 is not included in the JVAS catalogue, and in Table 1 we report the position found in the NVSS catalogue with about 1 arcsec accuracy.

The data reduction followed standard procedures (debiassing, flat fielding) in each filter within the National Optical Astronomical Observatory (NOAO) IRAF package. The photometry was calibrated by using the standard stars (Landolt 1992) in two fields (SAO110-503 and PG 2331+055A), observed each night at different air masses. Both nights had about the same good photometric quality, and the photometry has an accuracy of about $0.05 \mathrm{mag}$ in the $R, B$ and $V$ filters, $0.1 \mathrm{mag}$ in the $I$ filter.

All the magnitudes were corrected for galactic extinction according to Schlegel et al. (1998), while no correction was made for the atmospheric contribution, which was generally within the accuracy of the photometry mentioned above.

The seeing turned out to be quite stable in both nights ranging from 1.0 to 1.3 arcsec.

\section{Results}

\subsection{Optical identification}

The optical astrometry was derived from a number of GSC stars present in each field around the HFP source. The errors in this procedure have been estimated to be smaller than the uncertainty between the radio and optical reference frames, and therefore neglected. The optical identification was successful for all sources except J0037+0808.

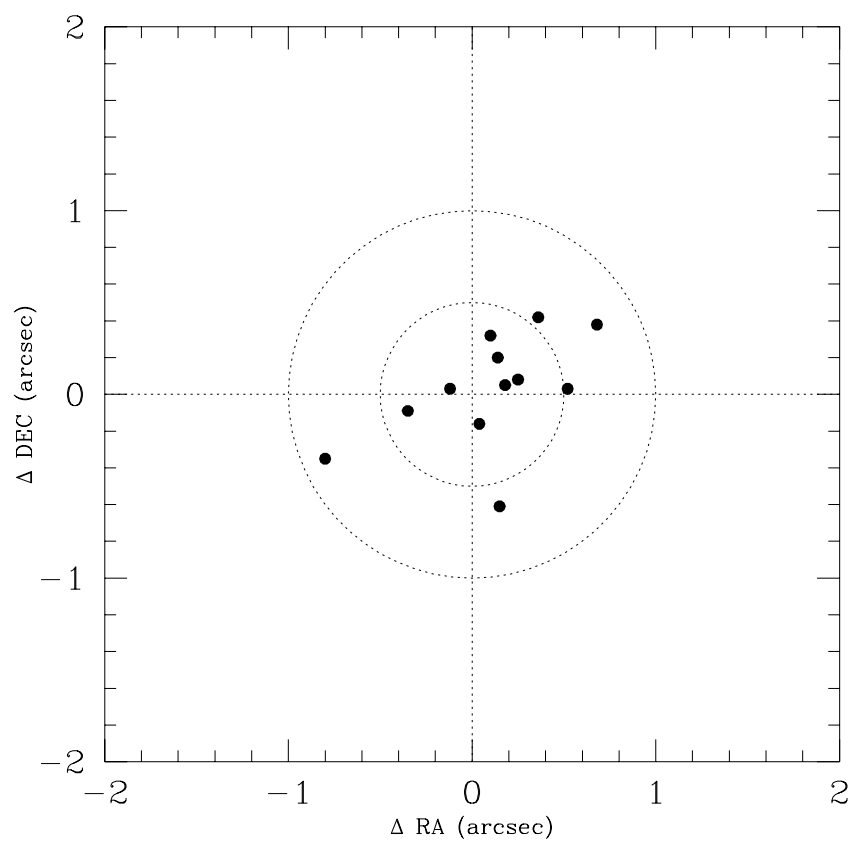

Fig. 1. Difference between RA and DEC derived from the optical and radio optical positions. The two dotted circles have radius of 0.5 and 1.0 arcsec.

A fit to the position of each host object was carried out in each filter. In order to have a homogeneous criterion, we decided to consider "optical position" the average of the RA and DEC obtained for the $R$ and $V$ images. The difference between these positions is generally within 0.1 arcsec. Two sources have slightly larger differences:

- J2021+0515: the host lies near a much brighter source (about $5^{\prime \prime}$, with $R=17.46$ ) and the $\sim 0.3^{\prime \prime}$ difference between the position in the two filters can be explained by the contamination from this source; 

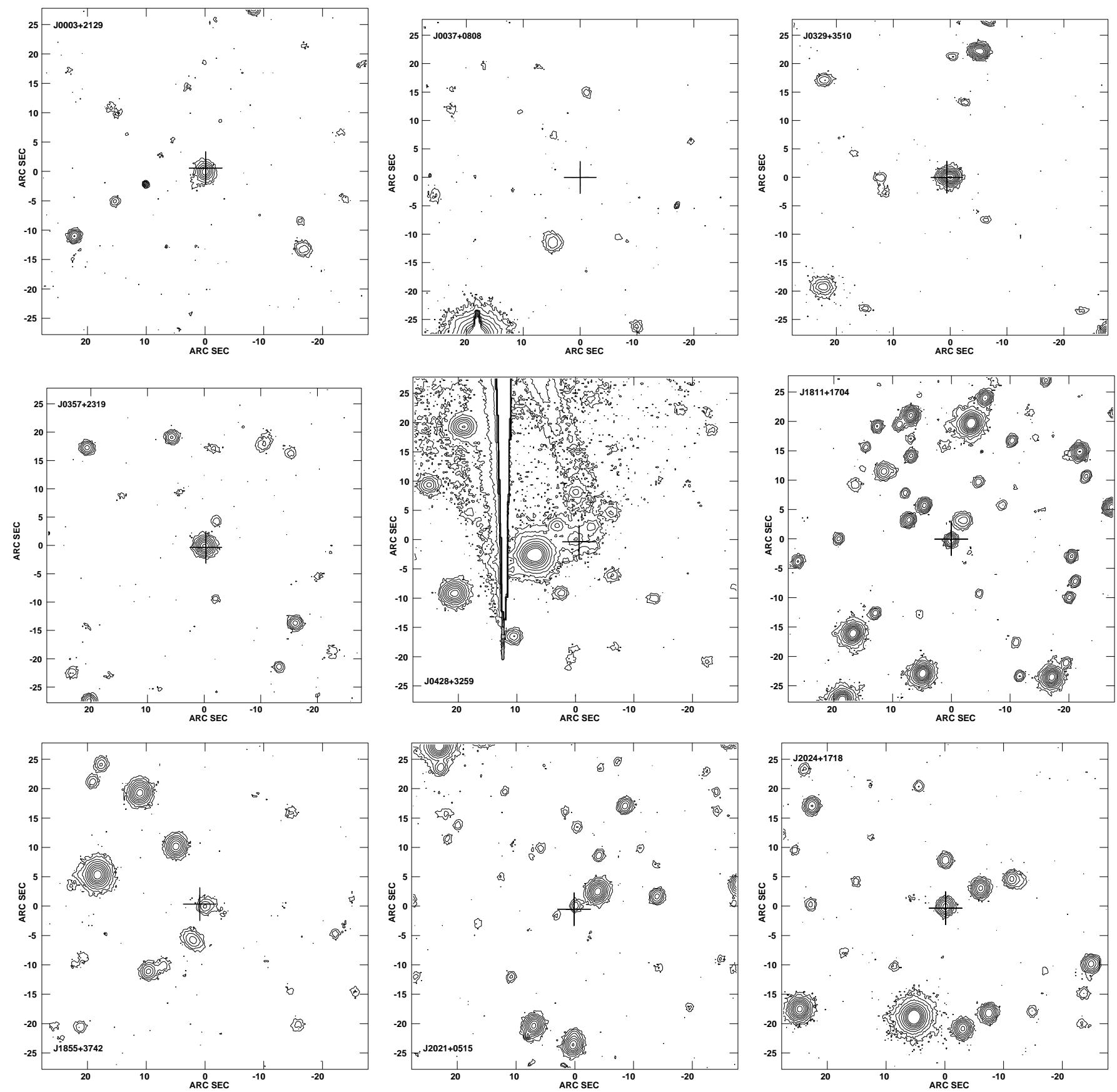

Fig. 2. $R$-band images of HFPs. Only the inner part of the CCD frame is presented. The HFP host is always at the center of the image, and the radio position is marked by a cross. North is up, East is to the left.

- J2203+1007: it is the weakest object identified in the present work, and its emission is clearly extended, without an outstanding peak, and partially contaminated by a nearby extended object, possibly a companion of the host of the HFP source.

We also measured the FWHM on the red images for all the 12 HFP hosts. The resulting angular sizes were then compared to the FWHM of stars in nearby regions in the same frame in order to estimate the extension of the HFP host and complement the information derived from the color index $(V-R)$. The classification was into two main classes, i.e. resolved ("R") or unresolved ("U") as reported in Col. 11 in Table 2. A question mark following the letter means that classification is uncertain.

\subsection{Notes on individual sources}

For a few sources we also comment on the POSS-II $(R$ and $B$ ) images, characterized by some increase in sensitivity with respect to the POSS-I $(R)$ images, when this information is relevant to outline the optical properties of the HFP host. 

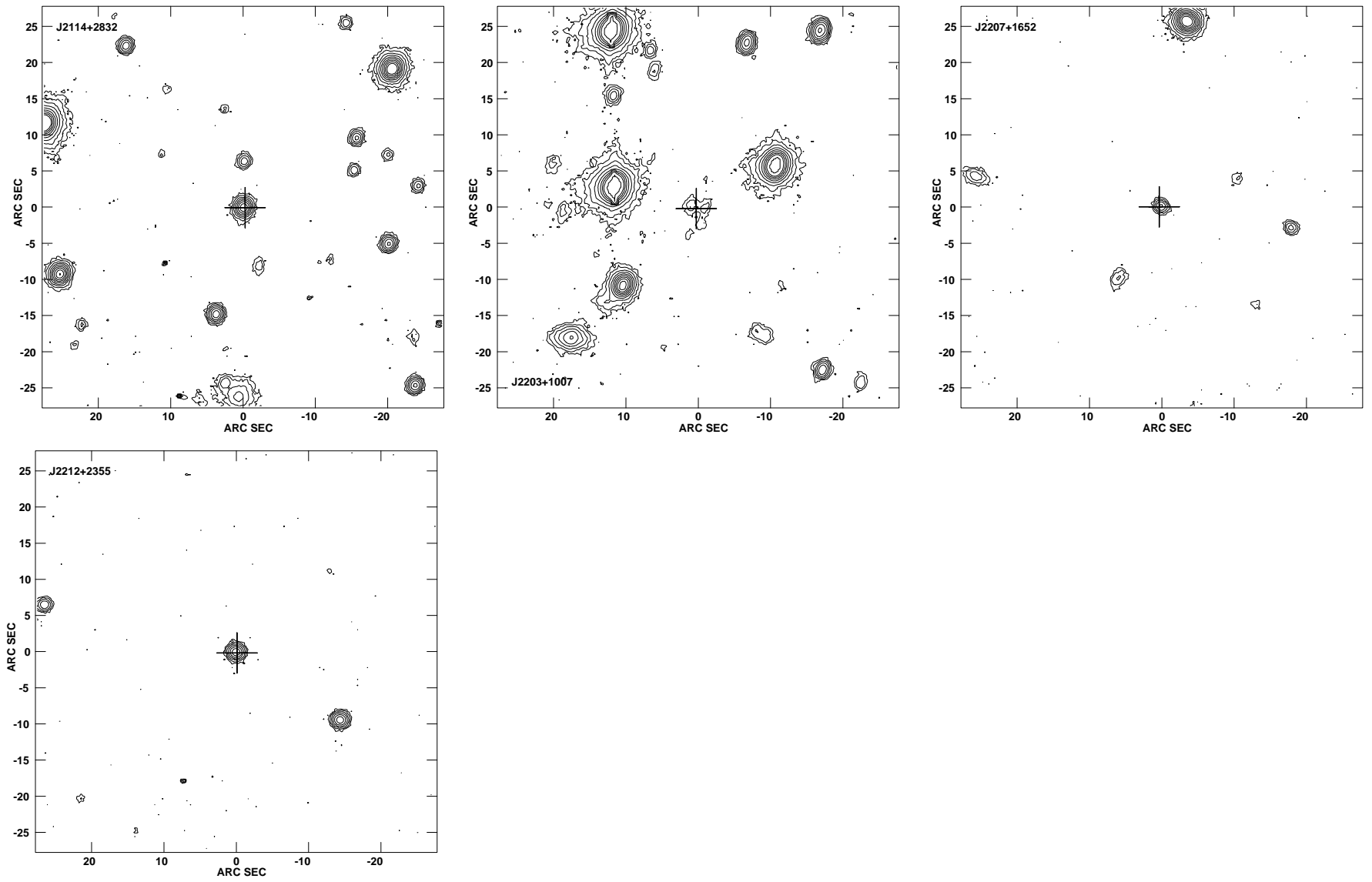

Fig. 2. continued.

$\mathrm{J} 0003+2129$

The radio source is coincident with the brightest optical object within $25^{\prime \prime}$ from the radio position. We measured $R=19.65$, and $V-R=0.93$. The lowest countour in Fig. 2 supports the hypothesis that this object is likely a galaxy. Indeed, also the FWHM measured for the HFP host is about $14 \%$ broader than that determined for the stars.

If we consider the Hubble diagram (Snellen et al. 1996), we can estimate a redshift of 0.4 . No optical counterpart is visible in the POSS-I image, while very weak emission is present in both $R$ and $B$ POSS-II images. It is likely that the optical emission of this object is variable, and has increased since the POSS-I and POSS-II observations.

\section{J0037+0808}

No significant emission has been detected in our images. No counterpart is visible in either POSS-I or POSS-II images. From the present observation, the upper limit to the $R$ magnitude is $m_{R}>24.5$. By assuming that the host is a galaxy and by using the forementioned relation we can set up a lower limit to the redshift of this object, likely to be at $z>1.8$.

\section{$\mathrm{J} 0329+3510$}

POSS-I and POSS-II images show a counterpart with stellar appearance. The TNG data confirm that the radio source is identified with a bright stellar object with a rather flat optical spectrum and with FWHM similar to those of the stars in the field. The $R$ band image in Fig. 2 is clearly affected by tracking problems, since all the point sources appear elongated approximately in E-W direction. There is a number of extended objects in the field in a narrow magnitude range about $4.5 \mathrm{mag}$ weaker than the HFP host.

\section{$J 0357+2319$}

The optical host is definitely a variable source. No counterpart can be found in the POSS-I images, while a weak object is visible in the POSS-II images just at the limit allowed by the sensitivity of the survey. Our observations revealed a relatively bright, unresolved object with $R=17.76$. There are various extended objects in the field around J0357+2319, spanning a narrow magnitude range about 4.5 mag weaker than the HFP host.

$$
\text { J0428+3259 }
$$

The optical identification is with an extended object of $R=19.25$ located very close to the radio position indicated by a cross in Fig. 2. The field is indeed rather complex, due to the presence of a number of nearby extended sources with magnitude similar to the HFP host or slightly weaker. The stray pattern of a bright star about $40^{\prime \prime}$ North of the radio source and another star about $7^{\prime \prime}$ to the West increase the local noise in the region around the HFP source.

If we refer to the Hubble diagram in Snellen et al. (1996) we can estimate a redshift of 0.3 for this galaxy. 
$J 1811+1704$

The optical field around the radio source is crowded by a number of objects with similar magnitude. The proposed optical identification is with an unresolved source sitting at about 0.1 arcsec from the radio position. There is an extended object 0.35 mag weaker at about $4^{\prime \prime}$ to the NorthEast. The HFP host is visible in the POSS-II red image, but it is not detected in the blue image.

$J 1855+3742$

The optical identification is with an object with $R=$ 20.70. Its FWHM is a few percent broader than the average value for the stars in this frame; in addition to this, some weak extended emission is visible West of the radio position, and possibly connected with the HFP host. An extended object with $R=20.29$ is located about $6^{\prime \prime}$ to the South. A weak emission is visible in both red and blue POSS-II images in the position of the HFP host, while no significant emission can be revealed in the less sensitive POSS-I red image. If we consider the HFP host a galaxy, its redshift can be estimated to be about 0.5 .

J2021+0515

No counterpart is visible either in POSS-I or in POSS-II images given the presence of a nearby stellar emission extending up to the radio position. Our TNG images have a better resolution (the seeing is about $1.2 \operatorname{arcsec}$ ) and the optical host of the HFP source is a rather weak object with $R=21.08$ whose FWHM is the same of the nearby stars. This object is carachterized by $V-R=-0.38$. A number of weak extended objects about $0.5-1 \mathrm{mag}(R)$ weaker than the HFP host are present in this field (see Fig. 2).

\section{J $2024+1718$}

This HFP radio source has an optical identification already, with a stellar object, whose redshift of 1.050 is reported without reference in the NED database. The comparison between the red and blue POSS-II images reveals that the HFP host is the bluest of the three objects in a line.

Our TNG data confirm the optical identification with an unresolved object with a rather flat optical spectrum.

$J 2114+2832$

A clear counterpart of stellar appearance is visible in all POSS images. Our TNG data confirm the optical identification is with an unresolved object with $R=18.35$ and a rather flat optical spectrum.

\section{$J 2203+1007$}

No counterpart is visible in any of the POSS images. The radio source position falls within two peaks of extended emission. We assume that the HFP host is the eastern object, being closer. Indeed there is some additional extended emission to the South, suggesting that there could be some interaction between galaxies.

This is the weakest object detected in this work, and is very likely to be a galaxy, given that $V-R=1.25$. Again, by using the Hubble diagram in Snellen et al. (1996) we can estimate the redshift for this source to be about 0.9 .
$J 2207+1652$

A weak counterpart is visible in all POSS images. The optical identification is with an unresolved source with $R=19.91$ and a rather flat optical spectrum. This source is somewhat weaker than the counterpart visible in the POSS-I and POSS-II images.

J $2212+2355$

The host of the radio source has a stellar profile and is much brigther in the $B$ filter than in $V$ and $R$. Also in the digitized POSS-II images the counterpart is significantly brighter in the blue than in the red plates.

\section{Summary and future work}

We have presented optical identifications for 12 out of 13 objects from a sample of 55 bright HFP radio sources. One of them has not been detected; it is likely a distant galaxy.

It turns out that five sources of the 13 observed (all empty fields on the POSS plates) can be classified as galaxies.

The remaining 8 objects, being unresolved in all our images and with rather blue colors, are likely distant quasars.

Based on the results of this paper and the identifications available in the literature only 12 out of the 46 HFPs with optical identification are associated to galaxies (26\%), and 34 to stellar objects (74\%).

Nine HFP sources do not have an optical identification yet (five are empty fields on the POSS plates). Assuming they have the same proportion between extended and unresolved morphologies found in these observations we expect that about half of them will be galaxies. This will raise the fraction of galaxies to about $30 \%$ This fraction is definitely lower than that in previous samples of bright CSS and GPS sources (Fanti et al. 1990; O'Dea 1998; Stanghellini et al. 1998).

Two HFP hosts, namely J0428+3259 and J2203+1007, appear to belong to small groups, with possible interaction, although further investigation to proof the physical connection with the other objects is required.

In a number of cases there are several weak galaxies with magnitudes distributed in a narrow range (0.5-1.0) located at small angular distances (within 30 arcsec) from the HFP host. However they are significantly weaker than the stellar HFP host, while they have comparable magnitudes in case of the galaxy J0428+3259.

Optical identification of the nine sources not yet imaged and redshift determination are needed in order to complete the classification and to derive the rest frame parameters of all sources.

Radio imaging with (sub-)milliarcsecond resolution in the optically thin region of the spectrum has been planned (VLBA time has been awarded) for the majority of the sources where the morphological information is missing or inadequate. Among these sources there are a few known Compact Symmetric Objects (CSOs), while a few more require confirmation. 
Acknowledgements. This research has made use of the NASA/IPAC Extragalactic Database (NED) which is operated by the Jet Propulsion Laboratory, California Institute of Technology, under contract with the National Aeronautics and Space Administration.

IRAF is distributed by KPNO, NOAO, operated by the AURA, Inc., for the National Science Foundation.

\section{References}

Baum, S. A., D'Dea, C. P., Murphy, D. W., \& de Bruyn, A. G. 1990, A\&A, 232, 19

Bicknell, G. V., Dopita, M. A., \& O’Dea, C. P. 1997, ApJ, 485, 112

van Breugel, W. J. M. 1984, in IAU Symp. 110, ed. R. Fanti, K. I. Kellerman, \& G. Setti (Reidel, Dordrecht), 59

Browne, I. W. A., Patnaik, A. P., Wilkison, P. N., \& Wrobel, J. M. 1998, MNRAS, 293, 257

Condon, J. J., Cotton, W. D., Greisen, E. W., et al. 1998, AJ, 115, 1693

Dallacasa, D., Stanghellini, C., Centonza, M., \& Fanti, R. 2000, A\&A, 363, 887

Fanti, C. 2000, Proc. 5th EVN Symp., ed. J. E. Conway, et al., 73

Fanti, C., Fanti, R., Dallacasa, D., et al. 1995, A\&A, 302, 317

Fanti, C., Pozzi, F., Fanti, R., et al. 2000, A\&A, 358, 499

Fanti, R., Fanti, C., Schilizzi, R. T., et al. 1990, A\&A, 231, 333

Gregory, P. C., Scott, W. K., Douglas, K., \& Condon, J. J.
1996, ApJS, 103, 427

Landolt, A. U. 1992, AJ, 104, 340

Murgia, M., Fanti, C., Fanti, R., et al. 1999, A\&A, 345, 769

O'Dea, C. P. 1998, PASP, 110, 493

Owsianik, I., Conway, J. E., \& Polatidis, A. G. 1998, A\&A, 336, L37

Owsianik, I, \& Conway, J. E. 1998, A\&A, 337, 69

Patnaik, A. R., Browne, I. W. A., Wilkinson, P. N., \& Wrobel, J. M. 1992, MNRAS, 254, 655

Readhead, A. C. S., Taylor, G. B., Pearson, T. J., \& Wilkinson, P. N. 1996, ApJ, 460, 634

Schlegel, D. J., Finkbeiner, D. P., \& Davis, M. 1998, ApJ, 500, 525

Snellen, I. A. G., Bremer, M. N., Schilizzi, R. T., Miley, G. K., \& van Ojik, R. 1996, MNRAS, 279, 1294

Snellen, I. A. G., Schilizzi, R. T., Bremer, M. N., et al. 1998, MNRAS, 301, 985

Snellen, I. A. G., Schilizzi, R. T., Miley, G. K., et al. 2000, MNRAS, 319, 445

Spencer, R. E., McDowell, J. C., Charlesworth, M., et al. 1990, MNRAS, 240, 657

Stanghellini, C., O'Dea, C. P., Baum, S. A., \& Laurikainen, E. 1993, ApJS, 88, 1

Stanghellini, C., O’Dea, C. P., Baum, S. A., et al. 1997, A\&A, 325, 943

Stanghellini, C., Dallacasa, D., O'Dea, C. P., et al. 2001, A\&A, 377, 377

Wilkinson, P. N., Browne, I. W. A., Patnaik, A. R., Wrobel, J. M., \& Sorathia, B. 1998, MNRAS, 300, 790 